\title{
A Feature-Based Approach for Loaded/Unloaded Drones Classification Exploiting micro-Doppler Signatures
}

\author{
Luca Pallotta \\ Dep. of Engineering \\ University of Roma Tre \\ Rome, Italy \\ luca.pallotta@uniroma3.it
}

\author{
Carmine Clemente \\ Dep. of Electronic and Electrical Eng. \\ University of Strathclyde \\ Glasgow, UK \\ carmine.clemente@strath.ac.uk
}

\author{
Alessandro Raddi \\ Dep. of Engineering \\ University of Roma Tre \\ Rome, Italy
}

ale.raddi@stud.uniroma3.it gaetano.giunta@uniroma3.it

\author{
Gaetano Giunta \\ Dep. of Engineering \\ University of Roma Tre \\ Rome, Italy
}

\begin{abstract}
This paper deals with the problem of loaded/unloaded drones classification. Precisely, exploiting the different micro-Doppler signatures exhibited by a drone with both any load and payloads of different weights, a novel signature extraction procedure is developed for automatic recognition purposes. The developed algorithms is based on a novel adaptation of the spectral kurtosis technique to the problem at hand, specifically the analysis of narrowband and wideband spectrograms of the radar echoes reflected by the drones. In addition, the principal component analysis is used to reduce the feature vector size. The experiments conducted on measured bistatic radar data prove the effectiveness of the proposed method in separating the quoted classes of objects.

Index Terms-micro-Doppler, automatic target recognition, drones classification, spectral kurtosis
\end{abstract}

\section{INTRODUCTION}

The past few years have seen a remarkable increase in interest in drone technology, ranging from drone deployment in urban areas to policy-making agendas. This is in turn largely due to the proliferation of these platforms becoming cheaper to buy off-the-shelf, or even to assemble in-house. However, attention from the media and concerns from law enforcement agencies and air traffic controllers have highlighted the potential misuse of these platforms, either accidental or voluntary: illegal filming; collision hazard with other aircraft at take-off and landing; transport of illicit material (e.g. smuggling drugs into prisons); weaponised drones. There is a clear awareness that more effective and sophisticated technologies are needed to detect, track and identify drones, manage their traffic, and make them detectable by authorities on the ground in order to mitigate possible hazards and threats. For radar sensors, small unmanned aerial vehicles (UAVs) represent an emerging class of targets for the following reasons: they exhibit low radar reflectivity due to small size and prevalence of plastic materials in their structure; they fly at low speed and altitude, confusing them with the static or slow-moving clutter in the Doppler domain; their highly maneuverable dynamics confuse

978-1-7281-8942-0/20/\$31.00 @2020 IEEE tracking algorithms that were designed for larger aircraft. Of particular interest is the capability to understand not only if the detected target is a drone or a different target, such as a bird or a vehicle in an antenna sidelobe, but also to identify variations of the configuration of a specific class of drones. Specifically, understanding whether a drone is equipped with a specific payload, or a payload of a specific weight can provide extremely useful insights on what and how dangerous the intentions of the drone could be. For instance, a drone used for non-professional application equipped with a camera only would have a much lighter payload than the same drone used to smuggle phones, weapons and drugs in a prison.

In recent years, a number of approaches have been applied to classify micro-Doppler signatures of drones and in general with targets with micro-motions. Approaches investigated features extracted from the received I/Q radar signal using empirical mode decomposition (EMD) [1] as well as extraction of features from both high resolution range profiles and Doppler [2]. The use of deep learning for the challenge of drones classification is also being investigated with approaches such as the one presented in [3] that demonstrated how multi-layers perceptron can provide high classification rates of drones with different propellers, while the method proposed in [4] has shown how deep learning can be useful to denoise the micro-Doppler signature before a classification stage. The work developed in [5], instead, has investigated how the micro-Doppler signature is affected by different weights of the payload and proposes additional strategies to classify the radar returns in a multi-static radar system. Moreover, the approach proposed in [6] exploit a novel track-beforedetect algorithm which jointly estimates the micro-Doppler signature, dominant reflectivity and kinematic trajectory of an object before the decision on its existence is made based on a constant false alarm rate (CFAR) detection scheme. The authors of [7] applied principal component analysis (PCA) to the time-frequency distributions of both direct-path and multi-path signals to extract meaningful features of the small UAVs that are successively fused. Finally, in [8], the authors developed a framework to detect and classify drones in a 
persistent range-Doppler radar. Following a CFAR detection stage, a convolutional neural network is used to perform the target recognition.

In this paper we introduce a new micro-Doppler signature extraction procedure based on the exploitation of the so-called spectral kurtosis [9]. This statistical tool has the intrinsic capability of emphasizing the non-stationary and/or non-Gaussian components in the frequency domain. As a matter of fact, since for Gaussian and stationary signals the spectral kurtosis assumes very low values, it significantly grows when nonstationary and non-Gaussian terms arise [10]. Thanks to these important characteristics, the spectral kurtosis has been utilized in several contexts; as an example it is worth to underline its application to the problem of vibratory monitoring of rotating machines [11]. From the above mentioned properties of the spectral kurtosis it follows the idea to apply and exploit this fourth-order statistical tool for classification purposes of signals derived by objects that are characterized by rotating parts as the drones.

The remainder of the paper is organized as follows. Section II introduces the definition of spectral kurtosis together with the description of the proposed feature extraction algorithm. The classification capabilities of the proposed technique are assessed in Section III, where some classification results on measured radar data of loaded/unloaded drones are shown. Finally, Section IV gives some conclusions and some hints for possible future works.

\section{Drone Classification Algorithm Based ON SPECTRAL KURTOSIS}

This section describes the proposed algorithm for drone classification based on micro-Doppler signature exploitation. More precisely, starting from the spectrogram of the acquired signal, the proposed procedure derives the so-called spectral kurtosis in order to emphasize and exploit the non-stationary or non-Gaussian components in the frequency domain. In the next subsections first of all the spectral kurtosis is introduced and described in details from a theoretical point of view, then the proposed feature extraction-based algorithm is shown.

\section{A. Spectral Kurtosis}

The concept of spectral kurtosis was firstly introduced in [9] to extract and observe additional information of non-Gaussian signals that would not be revealed by computing a classic power spectral density (PSD). As a matter of fact, the spectral kurtosis simply consists in computing the kurtosis for each frequency contained in the signal so as to emphasize its nonstationarities together with their corresponding location in the frequency domain. Therefore, the spectral kurtosis has been indicated by [9] as a statistical tool to be utilized in conjunction with the PSD to identify both non-stationary and/or nonGaussian components in frequency. This is confirmed by the fact that the spectral kurtosis assumes very low values for that frequencies in which the stationary Gaussian signals are located, whereas higher values in correspondence of nonstationarities [10].
Following the lead of [9], in [12] a simple definition of the spectral kurtosis utilizing the normalized fourth-order moment of the short-time Fourier transform (STFT) [13] magnitude has been provided. Precisely, indicating with $s(n), n=0, \ldots, N-$ 1 , a generic discrete-time signal comprising $N$ samples, its spectral kurtosis, say $\Psi(\nu)$, is proportional to the ratio between the fourth-order moment of its STFT and the square modulus of the second-order moment of its STFT, namely

$$
\Psi(\nu)=\frac{\frac{1}{K} \sum_{k=0}^{K-1}|\operatorname{STFT}(\nu, k)|^{4}}{\left(\frac{1}{K} \sum_{k=0}^{K-1}|\operatorname{STFT}(\nu, k)|^{2}\right)^{2}}-2,
$$

where $\operatorname{STFT}(\nu, k), k=0, \ldots, K-1$, is the $\operatorname{STFT}$ of $s(n)$, that is formally defined as

$$
\begin{gathered}
\operatorname{STFT}(\nu, k)=\sum_{n=0}^{N-1} s(n) w^{*}(n-k) e^{-j 2 \pi \nu n / N}, \\
k=0, \ldots, K-1,
\end{gathered}
$$

where $w(\cdot)$ is the smoothing window function used in the STFT whereas $\nu \in[-1 / 2,1 / 2]$ indicates the normalized frequency. It is now worth to recall that, in the subsequent sections, the spectral kurtosis in (1) is computed starting from the spectrogram, which is defined as the square modulus of the STFT. Finally, note that the frequency resolution in the spectrogram is inversely proportional to the temporal smoothing window size. As a consequence, also the spectral kurtosis depends on this parameter. However, further details on these aspects will be given in the next section.

\section{B. Feature Extraction Algorithm}

The proposed feature extraction algorithm based on microDoppler exploitation is graphically depicted in Figure 1.

As highlighted in the block-scheme, the proposed procedure starts with the computation of the spectrogram of the reference signal. As in [14]-[16] the spectrogram is chosen in place of alternative time-frequency distributions mainly for its robustness to interference [13]. More precisely, the spectrogram is computed twice for two smoothing windows of different size, say $w_{1}(\cdot)$ (of length $M_{1}$ ) and $w_{2}(\cdot)$ (of length $M_{2}$ ). This is done, since the second step is the evaluation of the spectral kurtosis that, as a consequence of the two considered smoothing windows, allows to extract different non-stationary components within the overall acquired signal, namely with a finer (coarser) frequency resolution. Indicating now, the vectors containing the values of the spectral kurtosis for all the considered normalized frequency values with $\psi_{1}=\left[\Psi_{1}(-1 / 2), \ldots, \Psi_{1}(1 / 2)\right]^{T}$ and $\boldsymbol{\psi}_{2}=\left[\Psi_{2}(-1 / 2), \ldots, \Psi_{2}(1 / 2)\right]^{T}$, respectively for the two corresponding smoothing functions, they are then lined up to form the extracted feature vector 


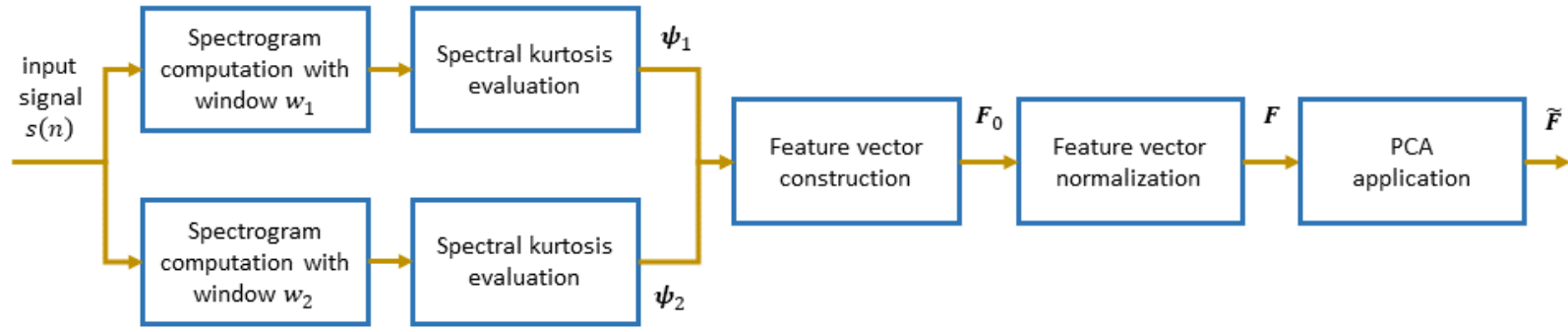

Figure 1. Block scheme of the proposed feature extraction algorithm.

$$
\boldsymbol{F}_{0}=\left[\begin{array}{l}
\boldsymbol{\psi}_{1} \\
\boldsymbol{\psi}_{2}
\end{array}\right] .
$$

The next step of the devised algorithm consists in normalizing the resulting feature vector, $\boldsymbol{F}_{0}$, thanks to the following linear rescaling

$$
\boldsymbol{F}=\frac{\boldsymbol{F}_{0}-\mu_{\boldsymbol{F}_{0}}}{\sigma_{\boldsymbol{F}_{0}}}
$$

where $\mu_{\boldsymbol{F}_{0}}$ is the mean and $\sigma_{\boldsymbol{F}_{0}}$ the standard deviation of the feature vector $\boldsymbol{F}_{0}$. This is done to avoid that a very strong feature value could polarize the decision of the classifier. Now, it is not difficult to observe that the above feature vector $\boldsymbol{F}$ contains a high number of elements that could reflect into a growth of the computational cost of the entire classification procedure. In addition, the values contained in $\boldsymbol{F}$ are not uncorrelated with each other, therefore a strong redundancy is present within it. A solution to solve these issues is given by the last step of the designed algorithm, in which the PCA is applied [17], [18]. More precisely, the PCA allows to reduce the dimension of the feature vector $\boldsymbol{F}$, indicated as $\tilde{\boldsymbol{F}}$ in the following, but at the same time it decorrelates its components producing also an increment in the discriminating capabilities of the proposed features. The classification is then performed using a k-nearest neighbour $(\mathrm{k}-\mathrm{NN})$ classifier for its low computational burden [19], with $\mathrm{k}$ set equal to 5 in the next analyses.

\section{TESTS AND RESUlts}

This section is aimed at showing the classification capabilities of the algorithm described in Section II in terms of drones discrimination exploiting their micro-Doppler signatures. Precisely, the analyses have been conducted on a measured dataset comprising loaded and unloaded drones collected with the multistatic radar system NetRAD of the University College London [20], [21]. The NetRAD system is a coherent pulsed radar composed by one transmitter (with a transmitting power of $+23 \mathrm{dBm}$, and horizontally polarized antennas with 24 $\mathrm{dBi}$ gain and approximately $10^{\circ} \times 10^{\circ}$ beamwidth) and three receivers operating in S-band (i.e., at $2.4 \mathrm{GHz}$ ) and sharing the same characteristics. The experiment for the acquisition of the data took place in July 2015 in an open football field at the UCL Sports Ground to the north of London, and the details of the conducted experiments are deeply described in [21]. However, for completeness the main characteristics of the NetRAD as set for the acquisition campaigns are summarized in Table I.

Table I

NETRAD PARAMETERS [21].

\begin{tabular}{cc}
\hline parameter & value \\
\hline \hline transmitted power & $23 \mathrm{dBm}$ \\
\hline antenna polarization & $\mathrm{HH}$ \\
\hline antenna gain & $24 \mathrm{dBi}$ \\
\hline antenna beamwidth & $10^{\circ} \times 10^{\circ}$ \\
\hline transmitted signal & linear up-chirp \\
\hline signal bandwith & $45 \mathrm{MHz}$ \\
\hline pulse duration & $0.6 \mu \mathrm{s}$ \\
\hline pulse repetition frequency (PRF) & $5 \mathrm{kHz}$ \\
\hline
\end{tabular}

In Figure 2 it is illustrated the setup configuration of the multistatic NetRAD for the experiments conducted for the acquisition of the dataset described in [21] and herein utilized to assess the performance of the proposed classification algorithm. From the scheme it is clear that the NetRAD is composed by a transmitter and three receiving nodes that are located on a baseline with a separation of $50 \mathrm{~m}$ from node to node. Moreover, the transmitter location is the same as the central receiver. The acquisitions have been done using as target a micro-drone (namely a quadcopter DJI Phantom Vision 2+) both hovering at $70 \mathrm{~m}$ and flying approximately from $90 \mathrm{~m}$ to $60 \mathrm{~m}$ from the baseline, with a consequent bistatic angle of approximately $40^{\circ}$.

For the acquisition, the camera installed on the micro-drone has been replaced with a plastic tray containing different payloads of small metallic disks, each weighting $10 \mathrm{~g}$. In this way, a total of six different classes of target has been obtained, viz., drone without payload (unloaded in the following) and drone with payload (referred to as loaded) of 200, 300, 400, 500 , and $600 \mathrm{~g}$, respectively. For each class a total of 45 acquisitions has been made with the respective signal of duration equal to $2 \mathrm{~s}$; moreover, as already said, both hovering and flying drones have been exploited to complete the tests, 


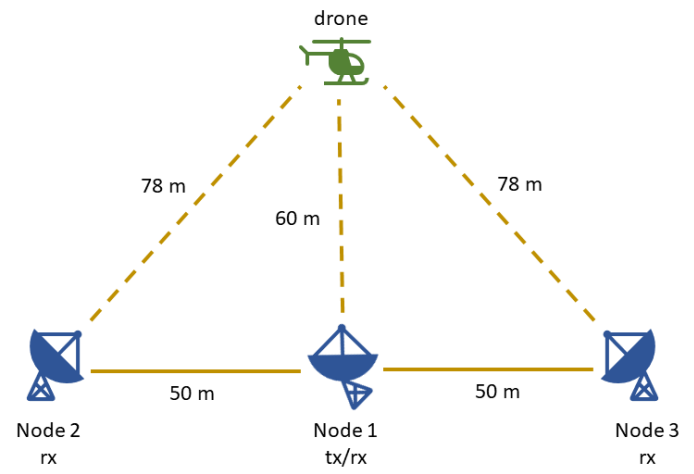

Figure 2. Setup configuration used for acquiring the measured data.

therefore a total of 90 acquisitions have been used. Figure 3 shows an example of spectrogram of an unloaded drone, subplot a), and a loaded hovering drone with a load of 500 g, subplot b), computed with $N_{\text {DFT }}=512$, Hamming window of size $M=500$ samples and overlap at $90 \%$. In addition, subplots c) and d) shows the related spectral kurtosis. Note that, the sizes of the two windows, $w_{1}(\cdot)$ and $w_{2}(\cdot)$, used to compute the spectral kurtosis in (3), have been set equal to $M_{1}=500$ and $M_{2}=125$ samples (corresponding to a duration of $0.1 \mathrm{~s}$ and $25 \mathrm{~ms}$, respectively), to calculate the features. Finally, the feature vector $\tilde{\boldsymbol{F}}$ has been reduced by a factor 16 with respect to $\boldsymbol{F}$ (i.e., passing from 1024 to 64 features).

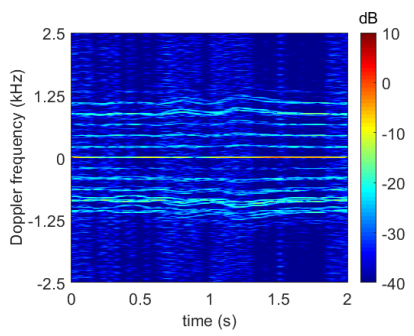

(a)

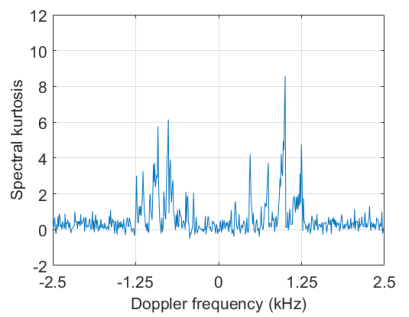

(c)

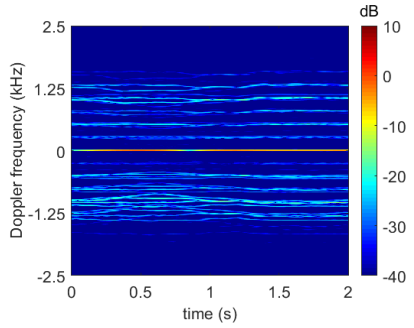

(b)

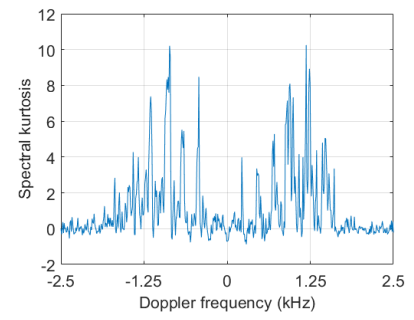

(d)
Figure 3. Spectrogram of a) unloaded and b) loaded (500 g) drone in hovering, and corresponding spectral kurtosis in c) and d), respectively.

The effectiveness of the proposed algorithm (synthetically referred to as SK in the following) has been assessed considering as figure of merit the correct classification defined as the ratio between the number of correct classified signals
Table II

$P_{c c}(\%)$ FOR THE SIX CLASSES OF DRONES

\begin{tabular}{cccc}
\hline & hovering and flying & hovering & flying \\
\hline \hline SK & $78.36 \%$ & $92.61 \%$ & $70.83 \%$ \\
\hline pZ & $73.19 \%$ & $92.05 \%$ & $55.64 \%$ \\
\hline Kr & $72.50 \%$ & $89.29 \%$ & $57.33 \%$ \\
\hline
\end{tabular}

over the total. More precisely, the tests have been performed dividing the data so as that the $70 \%$ are used for training, and the remainder $30 \%$ for the test phase. In addition, to provide a statistical characterization of the entire classification method, the average correct classification, indicated as $P_{c c}$, is computed following the classic Monte Carlo simulation procedure, namely 100 different experimental cases have been performed, randomly selecting the training and test sets of the data for each class. Finally, the results are shown also in comparison with the algorithm provided in [15] based on the exploitation of the pseudo-Zernike moments, say $\mathrm{pZ}$, and the technique of [22], [23] based on the extraction of the Krawtchouk moments (indicated as $\mathrm{Kr}$ in the following).

Table II shows the classification results in terms of $P_{c c}$ for the above defined six classes and considering the data related to jointly hovering and flying and also hovering and flying alone. From the table it is evident that, despite the flying case is the more challenging, the SK is still capable of ensuring satisfactory performance reaching the $70.83 \%$ of correct classification. Conversely, both $\mathrm{pZ}$ and $\mathrm{Kr}$ provide low correct classification percentages. In addition, also in the other cases, the SK tend to outperform its competitors.

To give further insights about the classification capabilities of the proposed algorithm, in Table III the average confusion matrix of the proposed algorithm for the six considered classes of drones in hovering is reported. From the observation of the confusion matrix it is not difficult to see that more challenging situations arise for the $300 \mathrm{~g}$ and $600 \mathrm{~g}$ loaded drones which share the lower classification values. It is not surprising that the $600 \mathrm{~g}$ loaded drone tends to be confused with the $500 \mathrm{~g}$ counterpart with an average error equal to $12.61 \%$ of all the trials.

The last analysis herein reported shows how the number of training data impacts on the final classification performances. To do this, the $P_{c c}$ has been computed for three different number of training data, viz., $30 \%, 50 \%$, and $70 \%$. As before, the remainder data have been used for the tests and the performances have been evaluated over a total of 100 Monte Carlo trials randomly selecting the training and test datasets. The consequent results are graphically represented in Figure 4 for the proposed algorithm again in comparison with $\mathrm{pZ}$ and $\mathrm{Kr}$ counterparts. Interestingly, the trend in the performance observed before is maintained also for a lower number of training data. Additionally, it is worth to be underlined the fact that the proposed algorithm is capable to ensure a sufficient level of discrimination among the six classes with a $P_{c c}>60 \%$ in the challenging flying case also when only the $30 \%$ of training 
Table III

AVERAGE CONFUSION MATRIX FOR THE SIX CLASSES OF DRONES IN HOVERING.

\begin{tabular}{cccccccc}
\hline & & \multicolumn{7}{c}{ predicted } \\
\hline \hline \multirow{5}{*}{ unloaded } & $\mathbf{2 0 0} \mathbf{g}$ & $\mathbf{3 0 0} \mathbf{g}$ & $\mathbf{4 0 0} \mathbf{g}$ & $\mathbf{5 0 0} \mathbf{g}$ & $\mathbf{6 0 0} \mathbf{g}$ \\
\cline { 2 - 8 } & unloaded & $\mathbf{9 7 . 3 1} \%$ & $0.15 \%$ & $0.23 \%$ & $2.15 \%$ & $0.16 \%$ & $0 \%$ \\
\cline { 2 - 8 } & $\mathbf{2 0 0} \mathbf{g}$ & $1.23 \%$ & $\mathbf{9 5 . 0 8} \%$ & $1.23 \%$ & $2.46 \%$ & $0 \%$ & $0 \%$ \\
\cline { 2 - 8 } & $\mathbf{3 0 0} \mathbf{g}$ & $4.46 \%$ & $6.77 \%$ & $\mathbf{8 8 . 1 6} \%$ & $0.23 \%$ & $0 \%$ & $0.38 \%$ \\
\cline { 2 - 8 } & $\mathbf{4 0 0} \mathbf{g}$ & $0.08 \%$ & $0 \%$ & $3.15 \%$ & $\mathbf{9 5 . 8 5} \%$ & $0.92 \%$ & $0 \%$ \\
\cline { 2 - 8 } & $\mathbf{5 0 0} \mathbf{g}$ & $0.08 \%$ & $1.15 \%$ & $0.15 \%$ & $0.77 \%$ & $\mathbf{9 7 . 3 1} \%$ & $0.54 \%$ \\
\hline $\mathbf{6 0 0} \mathbf{g}$ & $0.54 \%$ & $0 \%$ & $4.77 \%$ & $0.08 \%$ & $12.61 \%$ & $\mathbf{8 2 . 0 0} \%$ \\
\hline
\end{tabular}

data are used; conversely, the competitors drop below the $50 \%$.

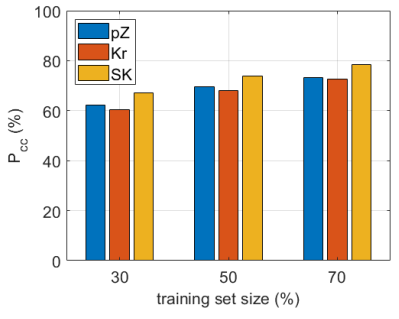

(a)

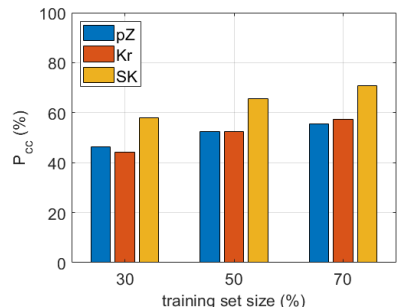

(b)

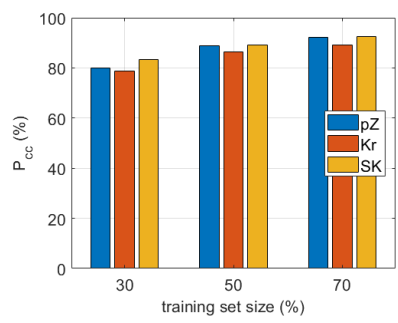

(c)

Figure 4. Average correct classification of six classes drones a) both flying and hovering, b) flying, and c) hovering.

\section{CONCLUSION}

In this paper we introduce a new micro-Doppler feature extraction procedure based on the exploitation of the socalled spectral kurtosis with the objective of UAV payload classification. The spectral kurtosis is computed from both the narrowband and wideband spectrograms obtained from the radar return and then is used as input to a classifier after a dimensionality reduction stage using PCA. The performance of the proposed feature are assessed on real radar data containing signatures of drones with different payloads with an average correct payload identification accuracy of $92.61 \%$. Future works could consist in evaluating the effect of different spectrogram windows lengths as well as different reduction factors in the PCA; moreover, the proposed approach could be also applied to distinguish between drones and birds. Finally, the study of a different feature selection approach could be also of interest.

\section{ACKNOWLEDGMENT}

The authors would like to acknowledge dr. Francesco Fioranelli and the University College of London for providing the real-recorded data.

\section{REFERENCES}

[1] Beom-Seok Oh, Xin Guo, Fangyuan Wan, Kar-Ann Toh, and Zhiping Lin, "Micro-Doppler Mini-UAV Classification using Empirical-Mode Decomposition Features," IEEE Geoscience and Remote Sensing Letters, vol. 15, no. 2, pp. 227-231, 2017.

[2] Jacco J. M. de Wit, Philip van Dorp, and Albert G. Huizing, "Classification of Air Targets Based on Range-Doppler Diagrams," in 2016 European Radar Conference (EuRAD). IEEE, 2016, pp. 89-92.

[3] Nir Regev, Ilia Yoffe, and Dov Wulich, "Classification of Single and Multi Propelled Miniature Drones Using Multilayer Perceptron Artificial Neural Network," in International Conference on Radar Systems (Radar). 2017, pp. 1-5, IET.

[4] Albert Huizing, Matijs Heiligers, Bastiaan Dekker, Jacco de Wit, Lorenzo Cifola, and Ronny Harmanny, "Deep Learning for Classification of Mini-UAVs Using Micro-Doppler Spectrograms in Cognitive Radar," IEEE Aerospace and Electronic Systems Magazine, vol. 34, no. 11 , pp. 46-56, 2019.

[5] Matthew Ritchie, Francesco Fioranelli, Hervé Borrion, and Hugh Griffiths, "Multistatic Micro-Doppler Radar Feature Extraction for Classification of Unloaded/Loaded Micro-Drones," IET Radar, Sonar \& Navigation, vol. 11, no. 1, pp. 116-124, 2016.

[6] K. Kim, M. Üney, and B. Mulgrew, "Coherent Track-Before-Detect with Micro-Doppler Signature Estimation in Array Radars," IET Radar, Sonar \& Navigation, vol. 14, no. 4, pp. 572-585, 2020.

[7] P. Zhang, G. Li, C. Huo, and H. Yin, "Exploitation of Multipath MicroDoppler Signatures for Drone Classification," IET Radar, Sonar \& Navigation, vol. 14, no. 4, pp. 586-592, 2020.

[8] I. Roldan, C. R. del Blanco, Á. D. de Quevedo, F. I. Urzaiz, J. G. Menoyo, A. A. López, D. Berjón, F. Jaureguizar, and N. García, "DopplerNet: a Convolutional Neural Network for Recognising Targets in Real Scenarios using a Persistent Range-Doppler Radar," IET Radar, Sonar \& Navigation, vol. 14, no. 4, pp. 593-600, 2020.

[9] R. Dwyer, "Detection of Non-Gaussian Signals by Frequency Domain Kurtosis Estimation," in ICASSP'83. IEEE International Conference on Acoustics, Speech, and Signal Processing. IEEE, 1983, vol. 8, pp. 607-610.

[10] J. Antoni, "The Spectral Kurtosis: A Useful Tool for Characterising Non-Stationary Signals," Mechanical Systems and Signal Processing, vol. 20 , no. 2 , pp. $282-307,2006$.

[11] J. Antoni and R. B. Randall, "The Spectral Kurtosis: Application to the Vibratory Surveillance and Diagnostics of Rotating Machines," Mechanical Systems and Signal Processing, vol. 20, no. 2, pp. 308331, 2006.

[12] C. Ottonello and S. Pagnan, "Modified Frequency Domain Kurtosis for Signal Processing," Electronics Letters, vol. 30, no. 14, pp. 1117-1118, 1994.

[13] L. Cohen, "Time-Frequency Distributions - A Review," Proceedings of the IEEE, vol. 77, no. 7, pp. 941-981, July 1989. 
[14] L. Pallotta, C. Clemente, A. De Maio, J. J. Soraghan, and A. Farina, "Pseudo-Zernike Moments based Radar micro-Doppler Classification," in 2014 IEEE Radar Conference, 2014, pp. 0850-0854.

[15] C. Clemente, L. Pallotta, A. De Maio, J. J. Soraghan, and A. Farina, "A Novel Algorithm for Radar Classification based on Doppler Characteristics Exploiting Orthogonal pseudo-Zernike Polynomials," IEEE Transactions on Aerospace and Electronic Systems, vol. 51, no. 1, pp. 417-430, 2015.

[16] A. R. Persico, C. Clemente, D. Gaglione, C. V. Ilioudis, J. Cao, L. Pallotta, A. De Maio, I. Proudler, and J. J. Soraghan, "On Model, Algorithms, and Experiment for Micro-Doppler-based Recognition of Ballistic Targets," IEEE Transactions on Aerospace and Electronic Systems, vol. 53, no. 3, pp. 1088-1108, 2017.

[17] S. Wold, K. Esbensen, and P. Geladi, "Principal Component Analysis," Chemometrics and Intelligent Laboratory Systems, vol. 2, no. 1-3, pp. 37-52, 1987.

[18] J. Zabalza, C. Clemente, G. Di Caterina, J. Ren, J. J. Soraghan, and S. Marshall, "Robust PCA Micro-Doppler Classification using SVM on Embedded Systems," IEEE Transactions on Aerospace and Electronic Systems, vol. 50, no. 3, pp. 2304-2310, 2014.

[19] R. O. Duda, P. E. Hart, et al., Pattern Classification and Scene Analysis, vol. 3, Wiley New York, 1973.

[20] F. Fioranelli, M. Ritchie, and H. Griffiths, "Multistatic Human MicroDoppler Classification of Armed/Unarmed Personnel," IET Radar, Sonar \& Navigation, vol. 9 , no. 7 , pp. 857-865, 2015.

[21] F. Fioranelli, M. Ritchie, H. Griffiths, and H. Borrion, "Classification of Loaded/Unloaded Micro-Drones using Multistatic Radar," Electronics Letters, vol. 51, no. 22, pp. 1813-1815, 2015.

[22] A. Aßmann, A. Izzo, and C. Clemente, "Efficient Micro-Doppler Based Pedestrian Activity Classification for ADAS Systems Using Krawtchouk Moments," in 11th IMA International Conference on Mathematics in Signal Processing, 2016.

[23] Adriano Rosario Persico, Carmine Clemente, Luca Pallotta, Antonio De Maio, and John Soraghan, "Micro-Doppler Classification of Ballistic Threats using Krawtchouk Moments," in 2016 IEEE Radar Conference (RadarConf). IEEE, 2016, pp. 1-6. 